\title{
The effect of feedback after good and poor trials on the continuous motor tasks learning
}

\author{
Reza Goudini' ${ }^{1}$, Esmaeel Saemi ${ }^{2, *}$, Saeed Ashrafpoornavaee ${ }^{1}$, and Behrouz Abdoli ${ }^{1}$ \\ ${ }^{1}$ Faculty of Sport Sciences, Shahid Beheshti University, Tehran, Iran; and ${ }^{2}$ Faculty of Sport Sciences, Shahid Chamran \\ University of Ahvaz, Ahvaz, Iran
}

Copyright: (c) 2018 R. Goudini et al. This is an open access article licensed under the Creative Commons Attribution License (http://creativecommons.org/licenses/by/4.0/).

\begin{abstract}
Background: Although the useful effects of motivational feedback on the motor skills learning have been proven, a study focusing on the effects of feedback after good and poor trials in continuous motor tasks has not been conducted. Objective: The purpose of the present study is to examine the effect of feedback after good versus poor trials on continuous motor tasks learning among novices. Methods: Eighteen right-handed students (age $24.66 \pm 1.35$ years) were randomly divided in two groups ( 7 males and 2 females each) of good trial and poor trials. All participants completed 11 blocks of 6 trials for a total of 66 practice trials in line-tracking task. After each block of 6 trials, participants in the "feedback after good trials" group received feedback on their 3 best trials in that block, whereas those in the "feedback after poor trials" group received feedback on their 3 poorest trials in the block. The practice was conducted in a day and a retention test was administered after 48 hours. Results: Our findings showed that feedback after good trials versus poor trials is significantly better for learning continuous motor tasks and performance $(p<.05)$. Conclusions: The findings of the present study highlight the motivational role of feedback in learning and performance of a continuous motor task.
\end{abstract}

Keywords: knowledge of results, good trials, poor trials, motivational feedback

\section{Introduction}

Among the variables influencing learning in physical exercises, augmented feedback (knowledge of result, knowledge of performance) plays a key role (Schmidt $\&$ Lee, 2005). Feedback can have many roles including the informational and motivational functions (Schmidt \& Wrisberg, 2008). In order to determine the content of feedback, a coach must pay attention to the aim of presenting information; if the aim is providing feedback for facilitating skill acquisition, the information related to the error must be presented; and if the aim is to confirm the encouragement to practice, s/he must underscore the characteristics of correct movement pattern (Coker, 2004).

Most of the experimental studies focused on the informational function of feedback which provides information about each person's performance in relation to the aim of the task. According to the guidance

\footnotetext{
* Address for correspondence: Esmaeel Saemi, Department of Sport Psychology, Faculty of Sport Sciences, Shahid Chamran University of Ahvaz, Golestan Blvd., Ahvaz, 61357-83151 Iran. E-mail: e.saemi@scu.ac.ir
}

hypothesis, the low frequency of the feedback and, in addition, feedback after poor trials and large errors when the learner is directed towards the correct movement pattern is considered to be more effective than feedback after good trials and small errors (Salmoni, Schmidt, \& Walter, 1984). However, recent studies in this area have provided real insights into the motivational role of the augmented feedback for motor learning (Badami, VaezMousavi, Wulf, \& Namazizadeh, 2012; Chiviacowsky \& Wulf, 2002; Saemi, Wulf, Varzaneh, \& Zarghami, 2011).The findings indicate that the motivational characteristics of feedback can have a direct effect on motor learning (Chiviacowsky \& Wulf, 2002, 2007; Chiviacowsky, Wulf, Wally, \& Borges, 2009). Taking into consideration the different approach to optimization of providing feedback in recent years' studies in physical exercises, providing feedback is based on the learner's demand which is called self-controlled feedback. Moreover, the research has shown that giving control to the learners in some situations during exercise and education enhances motor learning (Carter \& Patterson, 2012; Fairbrother, Laughlin, \& Nguyen, 2012; Wulf, 2007). 
Lai and Shea (1999) remarked that giving control to the learners during exercise makes the subject compare his/her good trials with the external-focus feedback provided by the examiner and, consequently, find successful performing strategies for them. Furthermore, feedback after good and poor trials is a type of self-controlled feedback which is provided for the best and worst trials based on the participants' demands. In most of the studies done in feedback after good and poor trials, such as an overhand throwing task (Chiviacowsky \& Wulf, 2002, 2007; Chiviacowsky et al., 2009; Saemi, Porter, Ghotbi-Varzaneh, Zarghami, \& Maleki, 2012) and Golf putting task (Badami, VaezMousavi, Wulf, \& Namazizadeh, 2011) were used. All these studies are a single task in which the movement plan is scheduled prior to performance. On the contrary, in continuous motor tasks schedule is of lesser significance and the priority is given to other factors, inter alia, feedback, detection and correction of errors which indicate more dependency on environmental stimuli (Schmidt \& Lee, 2005). Therefore, many aspects of performance and learning of single tasks and continuous tasks are different from each other (Schmidt \& Lee, 2005). By comparing the effect of self-controlled feedback and subject-controlled performance, Chiviacowsky and Wulf $(2002,2007)$ reached to the conclusion that most of the subjects demanded feedback after their good trials. In addition, the paired group with the self-controlled group had the same demand.

Moreover, Badami et al. (2011) and Ahmadi, Sabzi, Heirani, and Hasanvand (2011) pointed to the superiority of feedback after good trials over feedback after poor trials. The findings of Badami et al. (2012) also signified that the learning rate of the feedback group after fairly accurate trials in retention test was better than the other group. The studies which dealt with the effect of feedback after good trials on learning showed that feedback after good trial encourages the learners to achieve their goals and escalates their hope for the following performances (Salmoni et al., 1984).

Investigating the effect of motivational feedback in a self-controlled state started with the studies of Chiviacowsky and Wulf (2005) which indicated that motivational factors were responsible for self-controlled state and its resultant advantages. These results were repeated in the studies of Chiviacowsky et al. (2009), Saemi et al. (2012), and Badami et al. (2012) on the effect of feedback after good and successful trials. Since the studies done in good and poor trials utilized single tasks, we made use of continuous motor tasks to highlight the effects of feedback after good trials and poor trials on them.

Diverse studies are conducted in feedback after good and poor trials area (Badami et al., 2012;
Chiviacowsky \& Wulf, 2002, 2005, 2007; Chiviacowsky et al., 2009). They tackled providing motivation in people; but, a study focusing on the comparison of the effect of feedback after good and poor trials in a selfcontrolled state related to improving the performance and learning processes of continuous motor tasks has not been conducted yet. Unlike discrete skill tasks, continuous skill tasks need variables like feedback and error recognition (Schmidt \& Lee, 2005). Therefore, utilizing feedback can be influential on the improvement of learning and motor performance in continuous skill tasks; discrete skill tasks have been dealt with in previous studies (Badami et al., 2011; Saemi et al., 2012). However, it seems that feedback on successful and unsuccessful attempts in continuous skill tasks can provide us with more information about the mechanism of effectiveness concerning this type of feedback.

It seems that via analyzing the effectiveness of this kind of feedback, one can examine the effect of using different types of feedback after trials on the acquisition and retention of tasks and provide more accurate information. Thus, the aim of the present study is to examine the effect of feedback after good and poor trials on learning continuous motor tasks. Based on previous findings (e.g., Badami et al., 2011; Saemi et al., 2012), we predicted that providing feedback after good relative to poor trials could enhance motor performance in a continuous motor task.

\section{Methods}

\section{Participants}

Participants of this study consist of 22 right-handed students (age $24.66 \pm 1.35$ years), among whom four participants did not participate in the retention test, thus only 18 participants were further evaluated. They were randomly divided into two groups ( 7 males and 2 females each) of good trial and poor trial. The participants did not have any previous experience in carrying out the intended task. After completing an informed consent form, they took part in this research voluntarily. The study was approved by the university's institutional review board.

\section{Task}

The task contained doing line tracking (one hand) test from the subtests of MLS Motor Performance Series (The MLS is a modular test based on Fleishman's factor analysis of fine motor skills). A Vienna set was used to take the test (Vienna test system, Schuhfried, Vienna, Austria). In this task, participant should move a pen stylus in a groove without touching the walls of the groove. If the pen touches the groove's walls, the 
participant is corrected and then continues to move to the end of the groove. The track designed for the participants starts from the left side and ends in the right. The recorded variables include the number of errors (the number of times the walls of the groove were touched), the total duration of errors and the total time of movement.

\section{Procedure}

Participants were randomly assigned to either the "Knowledge of result (KR) after good trials" or the "KR after poor trials" group. All participants were informed that the task goal was to track the line (MLS) with their right hand. All participants completed 11 blocks of 6 trials for a total of 66 practice trials. After each block of 6 trials, participants in the "KR after good trials" group received KR on their 3 best trials in that block, whereas those in the "KR after poor trials" group received $\mathrm{KR}$ on their 3 poorest trials in the block. Before the initiation of the practice session, participants in both groups were informed that at the end of each block of 6 trials they would receive KR on three of the trials completed in the previous block. However, participants did not know if the provided KR was related to poor or good performances; rather they were informed that they were simply going to receive feedback about 3 attempts from the previous block of 6 trials. KR was presented to them for a few seconds. All participants returned after 48 hours and completed a 10-trial retention test; no KR was provided during the testing session.

Doing preliminary studies and examining their results, the time of each trial was taken to be $15 \mathrm{sec}$ onds with the interval of 10 seconds. Moreover, a twominute break between each trial and each block was examined as the criterion.

\section{Statistical analysis}

According to the normal distribution of the data, a 2 group (feedback after good and poor trials) $\times 11$ time (block order) mixed ANOVA with repeated measures on the last factor in the acquisition phase and the independent $t$-test in the retention test with the significance level of $p<.05$ were used. Effect size was reported as $\eta^{2}$ ( small $=.01$, average $=.06$, and large $=.14$ ) for Mixed ANOVA and Cohen's $d$ (small $=0.2$, average $=0.5$, and large $=0.8)$ for independent $t$-test (Cohen, 1988). SPSS was used for analysis of data (Version 16; SPSS, Chicago, IL, USA).

\section{Results}

\section{Acquisition phase}

The results of the $2 \times 11$ mixed ANOVA with repeated measures for mean number of errors, indicated that the main effect of practical blocks was significant, $\left.F(4.09,65.48)=11.65, p=.004, \eta^{2}=.042\right)$, and the main effect of groups was also significant $(F(1,16)=7.32$, $\left.p=.010, \eta^{2}=.031\right)$. The interaction between experimental groups and practical blocks were not significant. In regard of the duration of errors, results of the $2 \times 11$ mixed ANOVA with repeated measures indicated that the main effect of practical blocks was significant $\left(F(4.80,76.82)=12.68, p=.004, \eta^{2}=.044\right)$, and the main effect of experimental groups was also significant $\left(F(1,16)=4.01, p=.006, \eta^{2}=.210\right)$, however, the interaction between experimental groups and practical blocks were not significant. Furthermore, the results of the $2 \times 11$ mixed ANOVA with repeated measures for mean of total time indicated that the main effect of practical blocks was significant $(F(2.75,44.09)=3.50$, $\left.p=.02, \eta^{2}=.180\right)$, and also the main effect of experimental groups was significant $(F(1,16)=2.44, p=.013$, $\eta^{2}=.150$ ), however, the interaction between experimental groups and practical blocks were not significant (Figure 1, 2, and 3).

\section{Retention test}

The results of the independent $t$-test in the retention test indicated that there was a significant difference between the two experimental groups in the number of errors $(t(16)=-3.590, p=.002, d=0.6)$, and also the duration of errors $(t(12.25)=-5.421, p=.004$, $d=0.5$ ), on the other hand, the results of retention test indicated that feedback after good rather than

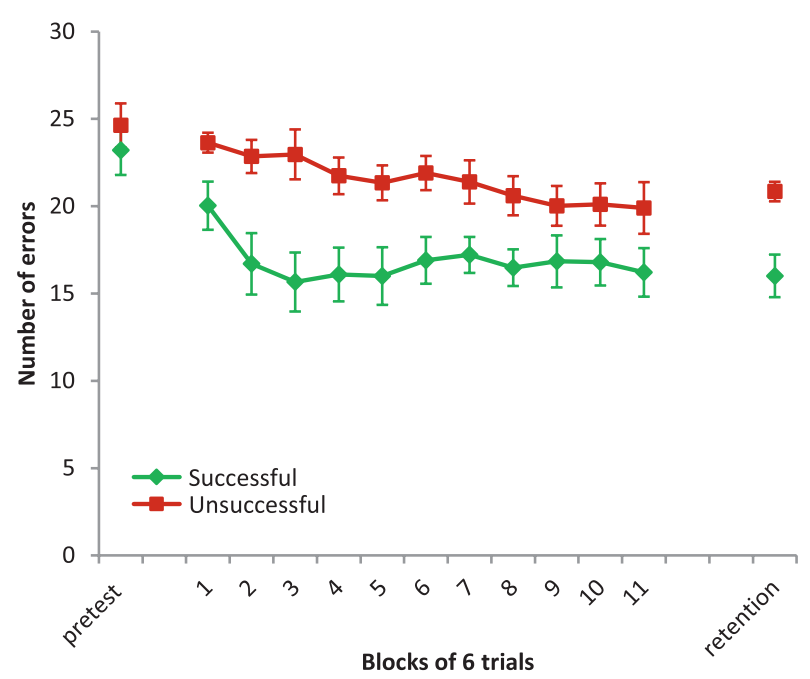

Figure 1. Number of errors (mean $\pm S D$ ) in the task of line tracking for each of the experimental conditions. 


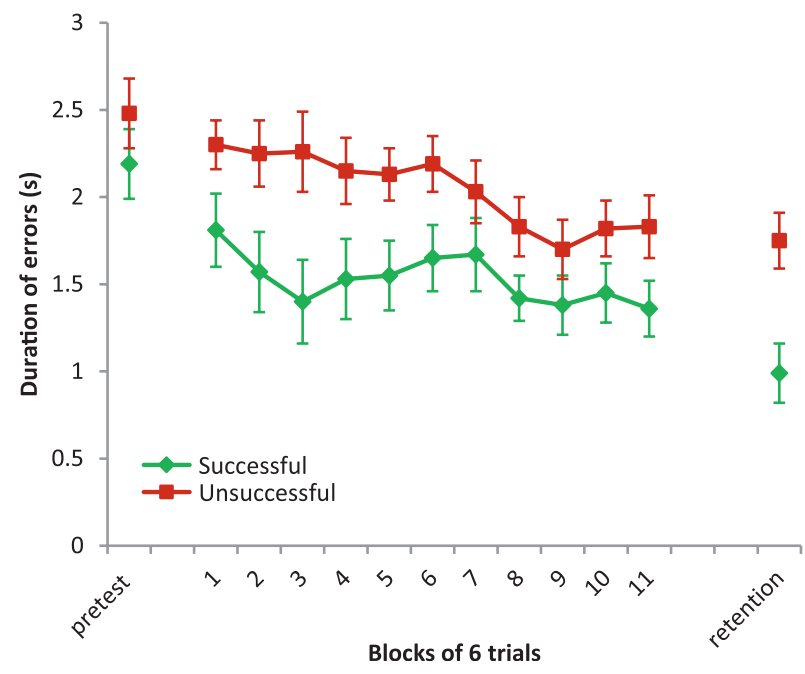

Figure 2. Duration of errors (mean $\pm S D$ ) in the task of line tracking for each of the experimental conditions.

poor trials had more beneficial on motor learning of the continuous motor task. However, for total time, no significant difference was found in retention test.

\section{Discussion}

The useful effects of feedback after good and poor trials on the learning of single tasks have been investigated in diverse studies; but, the effect of this kind of feedback on the continuous motor tasks has not been explored thus far. Therefore, the aim of the present study was to determine the effect of feedback after good and poor trials on the learning of a continuous motor task. The results of the present study showed that both groups had a significant improvement, however, the group with feedback after good trials rather than poor trials had better performance and motor learning on retention test. These results are in line with the previous findings (e.g., Badami et al., 2011; Chiviacowsky \& Wulf, 2002, 2005, 2007; Saemi et al., 2011). Taking into consideration the better performance of the group with feedback after good trials than the group with feedback after poor trials, one may deduce that feedback after good trials brings about a situation for encouraging the learners to repeat the good trials; hence, the emergence of a stable pattern. On the other hand, feedback after poor trials ends in the abortive variability of the response which is intended to correct the small errors. Consequently, this variability impedes the formation of a stable performance pattern. According to the evidence, repetition of a good performance pattern is easier than changing a performance pattern for correcting error. In addition, it leads to implicit learning (Chiviacowsky \& Wulf, 2007).

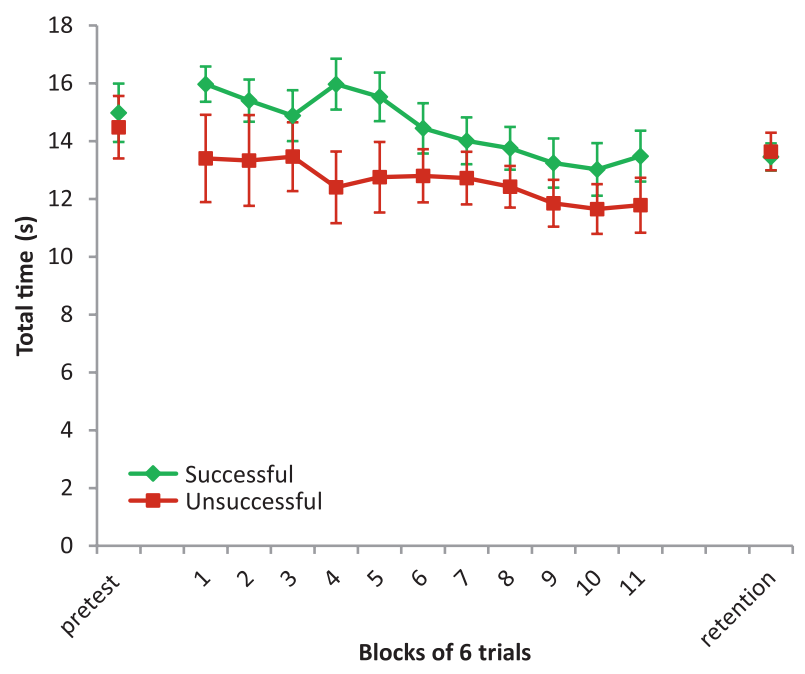

Figure 3. Total time (mean $\pm S D$ ) in the task of line tracking for each of the experimental conditions.

Furthermore, the findings the present study indicated that most of the participants tend to receive feedback after their good trials rather than their weak trials. These findings resonate with those of Chiviacowsky and Wulf (2005) and Badami et al. (2012). Feedback after good trials makes the subjects more enthusiastic to continue their performance. According to the results of comparing the group of good trial with the group of poor trial in the acquisition phase, the group of good trial was better than the group of poor trial in terms of hand-eye coordination. Since receiving feedback after good trials encourages the subjects to repeat more accurate performances, it seems that this issue reduces the variability of the response and brings about sort of scheme and the stability of perceptual trace. Consequently, this leads to the quick identification of the existing relationships among the components of the feedback (Guadagnoli \& Kohl, 2001). However, the noteworthy point in the current study and the studies done about the motivational feedback such as self-controlled feedback (Chiviacowsky \& Wulf, 2005; Chiviacowsky, Wulf, de Medeiros, Kaefer, \& Tani, 2008; Chiviacowsky, Wulf, Machado, \& Rydberg, 2012) and good and poor trials (Badami et al., 2012; Saemi et al., 2011) contained the lasting effect of this kind of feedback in the retention tests of doing tasks. The findings showed that those who received feedback after their good trials in the retention tests scored better than those who received feedback after their poor trials. These results confirmed the findings of Saemi et al. (2011), Badami et al. (2011), and Chiviacowsky et al. (2009). It seems that providing feedback after good trials instead of poor trials facilitates the learning of continuous tasks. This result is in line with the findings of Chiviacowsky and Wulf (2002, 
2007) and Chiviacowsky et al. (2009). According to the results of the research of Wulf, Chiviacowsky, and Lewthwaite (2012), a feedback which reduced tension in performance led to enhancement of learning. They related the enhancement of learning to using more of the process of self-control. Therefore, it is probable that feedback after good trials may need lesser attention in performance and the subject can use more of the process of self-control. But, when the feedback is provided for poor trials, it is probable that the subject may intentionally interfere in the process of self-control for the sake of correcting the errors and, by extension, his/her performance and learning scale down. Furthermore, based on the results of the present research in the retention test, the group of good trials had more hand-eye coordination and spatial timing. Probably, another factor influential in the superiority of the group with good trials over the group with poor trials is the subjects' tendency to receive feedback after their good trials (Badami et al., 2011; Chiviacowsky \& Wulf, 2005). The superiority of the group with feedback after good trials over the group with feedback after poor trials may originate from the motivational effect of feedback (Badami et al., 2012; Chiviacowsky \& Wulf, 2007) and the probable escalation of self-confidence in the group with the feedback after the good trials which left its effect in the retention test upon removal. Successful experience increase self-confidence and the beliefs which form in the subject on account of the kind of feedback anticipate the function (Fitzsimmons, Landers, Thomas, \& van der Mars, 1991). In addition, the learners of the group with feedback after good trial do not demand feedback randomly; rather, they have a specific strategy (Chiviacowsky \& Wulf, 2002). Thinking that they were approaching their goal, they demanded feedback which led to the escalation of the participants' sensitivity to how they function (Chiviacowsky \& Wulf, 2002). Those participants who received feedback positively and randomly witnessed some parts of their brain to be more active (Nieuwenhuis et al., 2005).

In the studies which dealt with the effect of feedback after good trials on learning, it became clear that feedback after good trials encourages the learners to achieve their goals and increases their hope for the following performances. The results of the research of Saemi et al. (2011) showed that the motivational consequences of feedback can have direct and positive effect on the children's skill learning and intrinsic motivation. Probably one of the reasons of the superiority of feedback after a good trial over feedback after a poor trial concerns the subjects' own tendency and demand. Moreover, the average time of doing the good trial was compared with that of the poor trial in the acquisition phase. The average time of doing the good trial (13.42 s) in the acquisition phase was higher than doing the poor trial $(12.73 \mathrm{~s})$ which shows that accuracy was more in the group of good trial. To justify these results, one can refer to the speed-accuracy trade-off of Fitts and Posner (1967), in which the escalation of accuracy leads to the reduction of number of errors. To put it differently, the more one strives for accuracy in doing the task, the more time is needed. On the other index, i.e., number of errors, we can observe the speed-accuracy trade-off of Fitts and Posner (1967), so that when the total time for doing the task decreased, proportionally the number of errors increased. Thus, it seems that feedback after good trials has a positive effect on accuracy in continuous motor tasks and paves the ground for the escalation of the subjects' motivation to repeat their successful experiences.

\section{Conclusions}

The findings of the present study indicated the motivational role of feedback even in learning of continuous motor tasks. The present findings have implications for coaches and practitioners, because of they tend to give feedback when their learners need it most to avoid errors, and to guide produced evidence of the motivational efficacy of feedback after good trials in contrast to poor trials.

\section{Conflict of interest}

There were no conflicts of interest.

\section{References}

Ahmadi, P., Sabzi, A. H., Heirani, A., \& Hasanvand, B. (2011). The effect of feedback after good, poor, good-poor trials, and self-control conditions in acquisition and learning of force production task. Scientific Journal Facta Universitatis, 9, 35-43.

Badami, R., VaezMousavi, M., Wulf, G., \& Namazizadeh, M. (2011). Feedback after good versus poor trials affects intrinsic motivation. Research Quarterly for Exercise and Sport, 82, 360-364.

Badami, R., VaezMousavi, M., Wulf, G., \& Namazizadeh, M. (2012). Feedback about more accurate versus less accurate trials: Differential effects on self-confidence and activation. Research Quarterly for Exercise and Sport, 83, 196-203.

Carter, M. J., \& Patterson, J. T. (2012). Self-controlled knowledge of results: Age-related differences in motor learning, strategies, and error detection. Human Movement Science, 31, 1459-1472. 
Chiviacowsky, S., \& Wulf, G. (2002). Self-controlled feedback: Does it enhance learning because performers get feedback when they need it? Research Quarterly for Exercise and Sport, 73, 408-415.

Chiviacowsky, S., \& Wulf, G. (2005). Self-controlled feedback is effective if it is based on the learner's performance. Research Quarterly for Exercise and Sport, 76, 42-48.

Chiviacowsky, S., \& Wulf, G. (2007). Feedback after good trials enhances learning. Research Quarterly for Exercise and Sport, 78, 40-47.

Chiviacowsky, S., Wulf, G., de Medeiros, F. L., Kaefer, A., \& Tani, G. (2008). Learning benefits of self-controlled knowledge of results in 10-year-old children. Research Quarterly for Exercise and Sport, 79, 405-410.

Chiviacowsky, S., Wulf, G., Machado, C., \& Rydberg, N. (2012). Self-controlled feedback enhances learning in adults with Down syndrome. Brazilian Journal of Physical Therapy, 16, 191-196.

Chiviacowsky, S., Wulf, G., Wally, R., \& Borges, T. (2009). Knowledge of results after good trials enhances learning in older adults. Research Quarterly for Exercise and Sport, $80,663-668$.

Cohen, J. (1988). Statistical power analysis for the behavioral sciences (2nd ed.). Hillsdale, NJ: Erlbaum.

Coker, C. A. (2004). Motor learning and control for practitioners. New York, NY: McGraw-Hill.

Fairbrother, J. T., Laughlin, D. D., \& Nguyen, T. V. (2012). Self-controlled feedback facilitates motor learning in both high and low activity individuals. Frontiers in Psychology, 3, 323 .

Fitts, P. M., \& Posner, M. I. (1967). Human performance. Belmont, CA: Brooks/Cole.

Fitzsimmons, P. A., Landers, D. M., Thomas, J. R., \& van der Mars, H. (1991). Does self-efficacy predict performance in experienced weightlifters? Research Quarterly for Exercise and Sport, 62, 424-431.
Guadagnoli, M. A., \& Kohl, R. M. (2001). Knowledge of results for motor learning: Relationship between error estimation and knowledge of results frequency. Journal of Motor Behavior, 33, 217-224.

Lai, Q., \& Shea, C. H. (1999). Bandwidth knowledge of results enhances generalized motor program learning. Research Quarterly for Exercise and Sport, 70, 79-83.

Nieuwenhuis, S., Slagter, H. A., Geusau, V., Alting, N. J., Heslenfeld, D. J., \& Holroyd, C. B. (2005). Knowing good from bad: Differential activation of human cortical areas by positive and negative outcomes. European Journal of Neuroscience, 21, 3161-3168.

Saemi, E., Porter, J. M., Ghotbi-Varzaneh, A., Zarghami, M., \& Maleki, F. (2012). Knowledge of results after relatively good trials enhances self-efficacy and motor learning. Psychology of Sport and Exercise, 13, 378-382.

Saemi, E., Wulf, G., Varzaneh, A. G., \& Zarghami, M. (2011). Feedback after good versus poor trials enhances motor learning in children. Revista Brasileira de Educação Física e Esporte, 25, 673-681.

Salmoni, A. W., Schmidt, R. A., \& Walter, C. B. (1984). Knowledge of results and motor learning: A review and critical reappraisal. Psychological Bulletin, 95, 355-386.

Schmidt, R. A., \& Lee, T. D. (2005). Motor control and learning (4th ed.). Champaign, IL: Human Kinetics.

Schmidt, R. A., \& Wrisberg, C. A. (2008). Motor learning and performance: A situation-based learning approach. Champaign, IL: Human Kinetics.

Wulf, G. (2007). Self-controlled practice enhances motor learning: Implications for physiotherapy. Physiotherapy, 93, 96-101.

Wulf, G., Chiviacowsky, S., \& Lewthwaite, R. (2012). Altering mindset can enhance motor learning in older adults. Psychology and Aging, 27, 14-21. 